\title{
Integrated analysis of whole-exome sequencing and transcriptome profiling in males with autism spectrum disorders
}

\author{
Marta Codina-Solà 1,2,3, Benjamín Rodríguez-Santiago ${ }^{4}$, Aïda Homs ${ }^{1,2,3}$, Javier Santoyo ${ }^{5}$, Maria Rigau', \\ Gemma Aznar-Laín ${ }^{6}$, Miguel del Campo ${ }^{1,3,7}$, Blanca Gener $^{8}$, Elisabeth Gabau ${ }^{9}$, María Pilar Botella ${ }^{10}$, \\ Armand Gutiérrez-Arumí ${ }^{1,2,3}$, Guillermo Antiñolo ${ }^{3,5,11}$, Luis Alberto Pérez-Jurado ${ }^{1,2,3^{*}}$ and Ivon Cuscó $1,2,3^{*}$
}

\begin{abstract}
Background: Autism spectrum disorders (ASD) are a group of neurodevelopmental disorders with high heritability. Recent findings support a highly heterogeneous and complex genetic etiology including rare de novo and inherited mutations or chromosomal rearrangements as well as double or multiple hits.

Methods: We performed whole-exome sequencing (WES) and blood cell transcriptome by RNAseq in a subset of male patients with idiopathic ASD $(n=36)$ in order to identify causative genes, transcriptomic alterations, and susceptibility variants.

Results: We detected likely monogenic causes in seven cases: five de novo (SCN2A, MED13L, KCNV1, CUL3, and PTEN) and two inherited X-linked variants (MAOA and CDKL5). Transcriptomic analyses allowed the identification of intronic causative mutations missed by the usual filtering of WES and revealed functional consequences of some rare mutations. These included aberrant transcripts (PTEN, POLR3C), deregulated expression in $1.7 \%$ of mutated genes (that is, SEMA6B, MECP2, ANK3, CREBBP), allele-specific expression (FUS, MTOR, TAF1C), and non-sense-mediated decay (RITI, ALG9). The analysis of rare inherited variants showed enrichment in relevant pathways such as the PI3K-Akt signaling and the axon guidance.

Conclusions: Integrative analysis of WES and blood RNAseq data has proven to be an efficient strategy to identify likely monogenic forms of ASD (19\% in our cohort), as well as additional rare inherited mutations that can contribute to ASD risk in a multifactorial manner. Blood transcriptomic data, besides validating $88 \%$ of expressed variants, allowed the identification of missed intronic mutations and revealed functional correlations of genetic variants, including changes in splicing, expression levels, and allelic expression.
\end{abstract}

Keywords: ASD, Whole-exome sequencing, CNV, SNV

\section{Background}

Autism spectrum disorders (ASD) [OMIM 209850] are defined as a group of neurobehavioral syndromes characterized by deficits in social interaction, impaired communication skills and restricted, stereotypical and ritualized patterns of interests and behavior, typically appearing before the age of 3. Throughout the last decades, the prevalence of ASD has risen from the historically estimated

\footnotetext{
*Correspondence: luis.perez@upf.edu; ivon.cusco@upf.edu 'Department of Experimental and Health Sciences, Universitat Pompeu Fabra, C/Doctor Aiguader 88, 422, Barcelona 08003, Spain

Full list of author information is available at the end of the article
}

proportion of $4 / 10,000$ to approximately $1 / 110$ children (2008) [1-3] with a ratio four times higher in males than females [4]. It is still debated how much of this increase is related to diagnostic improvements, raised awareness towards ASD, or emerging environmental factors $[5,6]$. ASD are among the most heritable neuropsychiatric disorders, given that concordance rates in monozygotic twins are $90 \%$ and siblings have an approximately 50 -fold increased risk of ASD. ASD are found in association with comorbid genetic conditions in 10\% of cases and are considered complex multifactorial disorders involving multiple genes 
$[7,8]$. Currently, the etiology can be established in only $30 \%$ of the cases and remains unknown for most patients.

The technological improvements of the last decade have lead to tremendous advances in understanding the genetic basis of ASD, revealing a high degree of genetic heterogeneity. Clinical application of molecular karyotyping has shown that $5 \%$ to $10 \%$ of patients carry chromosomal rearrangements and that the burden of rare and de novo smaller copy number variants (CNVs) is higher among ASD patients than controls. However, since many of these variants show incomplete penetrance and variable phenotypic expression, the best model to explain most ASD cases would be oligogenic with a probable environmental contribution. Until now, most genomic studies on ASD using next-generation sequencing (NGS) have focused on coding regions and have analyzed trios in an effort to identify de novo mutations [9-14]. Only a few studies investigated rare inherited variation [15-18]. The reported data suggest a contribution of de novo disruptive mutations in the genetic etiology of ASD, with hundreds of genes implicated and only a few of them recurrently mutated in unrelated cases (CHD8 [MIM 610528], DYRK1A [MIM 600855], GRIN2B [MIM 138252], KATNAL2 [MIM 614697], POGZ [MIM 614787], and SCN2A [MIM 182390]). Besides de novo disruptive mutations, comparison of rates of rare variation across the whole genome in cases versus controls have yielded no significant associations. These findings support previous hypothesis which suggested that a large number of genes confer risk to ASD and reinforce the idea that much larger cohorts will be necessary to carry out this type of analyses [19]. The identification of new genes involved in ASD will eventually lead to the definition of common effects of genetic variants and possibly ASD biomarkers and biological signatures. Biology system tools such as interaction networks are important to detect common deregulated pathways and expression networks implicated in the disease.

An additional approach to identify genetic variants associated with a phenotype and to understand the biological effects resulting from rare genetic variation could be derived from observing the transcriptomic consequences of genetic variation [20]. To this end, we have analyzed 36 Spanish male patients with idiopathic ASD by wholeexome sequencing (WES) to define causative or susceptibility variants for ASD and their transcriptomic consequences by RNAseq. In addition to the identification of likely monogenic cases, we also studied the accumulation of rare genetic variation which could result in putatively common functional consequences.

\section{Methods}

Sample selection

We studied 36 unrelated males with a diagnosis of idiopathic ASD selected from a Spanish cohort of 324 patients.
All cases except two were sporadic. All patients had a confirmed diagnosis of one of the categories of ASD listed in the Diagnosis and Statistical Manual of Mental Diseases IV (DSM-IV), categorized according to the Spanish version of ADI-R (Autism Diagnostic Interview-Revised), and the Wechsler Intelligence Scale for Children or Wechsler Adult Intelligence Scale. All patients had an extensive clinical and molecular evaluation including fragile $\mathrm{X}$ testing and molecular karyotype (either BAC, oligo, or SNP array) with normal results. The study was approved by the Clinical Research Ethics Committee of the centers involved (CEIC-Parc Salut Mar), and informed consent for participation was obtained from the parents or legal caregivers. Blood samples were obtained, and genomic DNA was extracted by the salting out method using the Puregene $^{\circ}$ DNA Purification Kit (Gentra Systems, Big Lake, MN, USA). Parental and familial samples were obtained from the available relatives who gave informed consent.

\section{Whole-exome capture and sequencing}

The exome portion of the genome was enriched using NimbleGen EZ Exome V2.0 capture kit (Roche Applied Science, Madison, WI, USA). Gene and exon annotations for SeqCap EZ Human Exome Library came from RefSeq (Jan 2010), CCDS (Sept 2009), and miRBase (v.14, September 2009). A total of approximately 30,000 coding genes (approximately 300,000 exons, total size $36.5 \mathrm{Mb}$ ) were targeted by the design, and a total of 44.1 $\mathrm{Mb}$ were covered by the probes. Final libraries were then sequenced on an ABI Solid 4 platform (Life Technologies, Carlsbad, CA, USA). Single-end sequences were obtained with a read length of $50 \mathrm{bp}$.

\section{Variant calling, annotation, and prioritization}

A pipeline for data alignment using BFAST [21] and GATK [22] algorithms was applied to the sequencing data following standard parameters. Briefly, sequences were aligned to the latest version of the human genome (hg19), PCR duplicates were marked and removed, and quality scores of alignments were recalibrated. Single nucleotide variants $(\mathrm{SNV})$ and indel calls were only considered if positions had a depth of coverage of at least $10 \times$, and heterozygous positions were only called when a minimum of $20 \%$ of the reads showed the variant ( $\mathrm{AB}$ between 0.2 and 0.8 ). In order to minimize technical artifacts, we removed variants that appeared in more than two samples, even if they were present in a single read or had an $A B$ ratio lower than 0.2. Annotation of variants was performed using ANNOVAR (http://www.openbioinformatics.org/annovar/), taking into account the variant frequency in control databases: dbSNP135 (http://www.ncbi.nlm.nih.gov/SNP/), Exome Variant Server (EVS) (http://evs.gs.washington.edu/EVS/), 
and an in-house database of 90 Spanish controls. The nature of the changes was assessed by PolyPhen and Condel (http://bg.upf.edu/fannsdb/) protein effect prediction algorithms [23]. To distinguish the putative disease-causing variants, we established the following criteria: (1) we selected only non-synonymous variants; (2) under a dominant model, we excluded variants previously described in the general population (dbSNP135, EVS, 1000 Genomes (http://browser.1000genomes.org) and Spanish controls); (3) under a recessive model, we removed variants with a minor allele frequency (MAF) $>0.002$ and only considered genes with homozygous or compound heterozygous mutations; (4) we discarded variants present in loss of function tolerant genes as previously described [24]; and (5) we manually inspected recurrent variants and indel calls to exclude false positives using Integrative Genomics Viewer (IGV) [25].

We used the XHMM algorithm to call CNVs, based on measurement of the read depth per target region (GATK). We followed the standard steps as described in the online tutorial. We applied the same filters previously described [26]: XHMM quality score (SQ) $\geq 65$, exons spanned $\geq 3$, and estimated CNV length $\geq 1 \mathrm{kB}$. We focused our analysis on rare CNVs, so we excluded CNVs overlapping with polymorphic variants reported in Database of Genomic Variants (DGV) (http://dgv.tcag.ca/dgv/app/home).

\section{Validation}

We used Sequenom genotyping (iPLEX Gold platform, San Diego, CA, USA) and Sanger sequencing by capillary electrophoresis (ABI PRISM 7900HT, Applied Biosystems, Foster City, CA, USA) to perform validation and segregation studies. To genotype the selected variants, we designed primers (PRIMER 3 application) (http://www.bio informatics.nl/cgi-bin/primer3plus/primer3plus.cgi/) and used standard PCR conditions. For CNV validation, we used multiple ligation probe amplification (MLPA) with custom probes in the target region. MLPA reactions were carried out under standard conditions. The relative peak height method was used to determine the copy number status. We analyzed samples from the proband and both parents as well as from other relatives when available.

\section{Paternity testing}

We performed microsatellite genotyping of trios to corroborate paternity on patients with de novo mutations. We selected highly heterozygous microsatellites markers randomly distributed in different autosomal chromosomes. PCR products were amplified under standard conditions, and fragments were separated and analyzed by high-resolution electrophoresis using GeneMapper software (ABI 3100, Applied Biosystems, Foster City, CA, USA).

\section{$\mathrm{X}$-chromosome inactivation analysis}

To determine the $\mathrm{X}$-chromosome inactivation pattern (XCI), we examined the differential methylation state of nearby HpaII sites of the polymorphic CAG repeat in exon 1 of the human androgen receptor gene (AR [MIM 313700]) located at Xq13. Following digestion with methylation sensitive restriction endonuclease HpaII, the region was amplified by PCR with a FAM labeled forward primer $[27,28]$. The digested and not digested PCR products were analyzed in an ABI PRISM 3100 Genetic Analyzer. For quantitative analysis, trace data were retrieved using the accompanying software (GeneMapper, Applied Biosystems, Foster City, CA, USA). The degree of XCI skewing was calculated as the fractional peak height ratio (expressed as \%) for the more strongly amplified allele. XCI was considered significantly skewed if the ratio exceeded 90:10.

\section{Transcriptome sequencing}

Peripheral mononuclear cells (PBMCs) from whole blood of 36 studied ASD patients were isolated using a ficoll density gradient (Lymphoprep ${ }^{\mathrm{Tw}}$, STEMCELL Technologies, Vancouver, British Columbia, Canada). Total RNA was extracted using Trizol (Life Technologies, Carlsbad, CA, USA) following a standard protocol. The quality and yield of the isolated RNA was assessed using a NanoDrop8000 Spectrophotometer (Thermo Fisher Scientific, Waltham, MA, USA) and Agilent 2100 Bioanalyzer (Agilent Technologies, Santa Clara, CA, USA). Transcriptome sequencing was performed on a HiSeq 2000. Paired-end sequences were obtained at a read length of 100 bp with 57,792,576 mean read pairs per sample. Sequences were aligned to the NCBI build 37 human genome reference using TopHat [29] and Bowtie [30] to map the inter-exon splice junctions. Cufflinks [31] and htseqcount [32] were used to estimate the expression of the transcripts (FPKM - fragment per kilobase of transcript per million fragments mapped- and read counts). We used the ComBat algorithm (http://www.bu.edu/jlab/ wp-assets/ComBat/Abstract.html) (package sva R) to remove batch effect and to obtain the z-score of expressed genes.

\section{Allele-specific expression analysis}

In order to study extreme imbalances of allelic expression, either allele-specific or preferential expression, we selected heterozygous SNPs (dbSNP135) identified by WES in each patient with a minimum depth of coverage of 15 and an $A B$ ratio between 0.3 and 0.7. SNPs in known segmental duplications or pseudogenes according to UCSC hg19 (http://genome.ucsc.edu/cgi-bin/hgGateway) 'Segmental Dups' and 'Retroposed Genes' tracks were excluded from the analyses. We then extracted the number of RNAseq reads mapped to each position and 
selected only highly covered positions (at least $20 \times$ ). We classified each SNP expression according to its $A B$ ratio, being biallelic when the $\mathrm{AB}$ ratio was between 0.1 and 0.9 and monoallelic when predominantly the reference or alternative allele were expressed $(\mathrm{AB}>0.9$ or $<0.1)$. When all SNPs of a gene were monoallelic, we classified the gene as having monoallelic expression, whereas genes with biallelic SNPs were considered to have biallelic expression.

\section{Isoform-splicing analysis}

The aligned reads were processed by Cufflinks, using a supplied reference annotation (Homo_sapiens.GRCh37.68.gtf) to guide RABT assembly. Assembled transcripts were then analyzed by Cuffcompare to compare isoforms across all samples. We then selected novel isoforms (defined by Cufflinks by class code $\mathrm{j}$ ) with an expression $>2$ FPKM and matched them to rare variants found by exome sequencing in the same patient.

\section{RNA editing analysis}

We first applied stringent filtering criteria to remove RNAseq false positive calls (DP $>10, \mathrm{SB} \leq 0.1$, HRun $<8$, ReadposRankSum $\geq 2.0$, BaseQRankSum $\geq 2$ ) and then annotated depth of coverage of the same positions according to exome sequencing. We selected only positions with a depth of coverage of at least $15 \times$ and that were not called by exome sequencing, excluding all variants described in control databases (dbSNP135, Exome Variant Server) and those present in another sample. We manually revised the remaining variants to discard false positive calls.

\section{Pathway enrichment analysis}

To identify common deregulated mechanisms affected by rare genetic variants, we performed pathway enrichment analyses using the publicly available ConsensusPathDB database (CPDB) (http://cpdb.molgen.mpg.de/). $\mathrm{CPDB}$ incorporates interaction data from different categories including metabolic and signaling reactions, physical protein and genetic interactions, or gene regulatory interactions. Statistical analyses were performed using the CPDB overrepresentation analysis option, with four categories of predefined genes (network neighborhood-based, pathways-based, Gene Ontology-based and protein complex-based gene sets). For each of the predefined sets, a $P$-value was calculated according to the hypergeometric test based on the number of physical entities present in both the predefined set and user-specified list of physical entities. For pathway-based sets, we used the default $P$ value threshold of 0.01 . We used the default gene background defined by CPDB as the number of entities that are annotated within the category of the provided gene. We then compared overrepresented pathways among rare WES variants in ASD samples with respect to 55 Spanish non-ASD samples.

\section{Results}

In order to perform the integrative analysis of nextgeneration sequencing data, we followed the workflow shown in Figure 1.

\section{WES general overview}

Overall, a mean depth of coverage of $40 \times$ was obtained and an average of 26,605 variants per sample was observed.

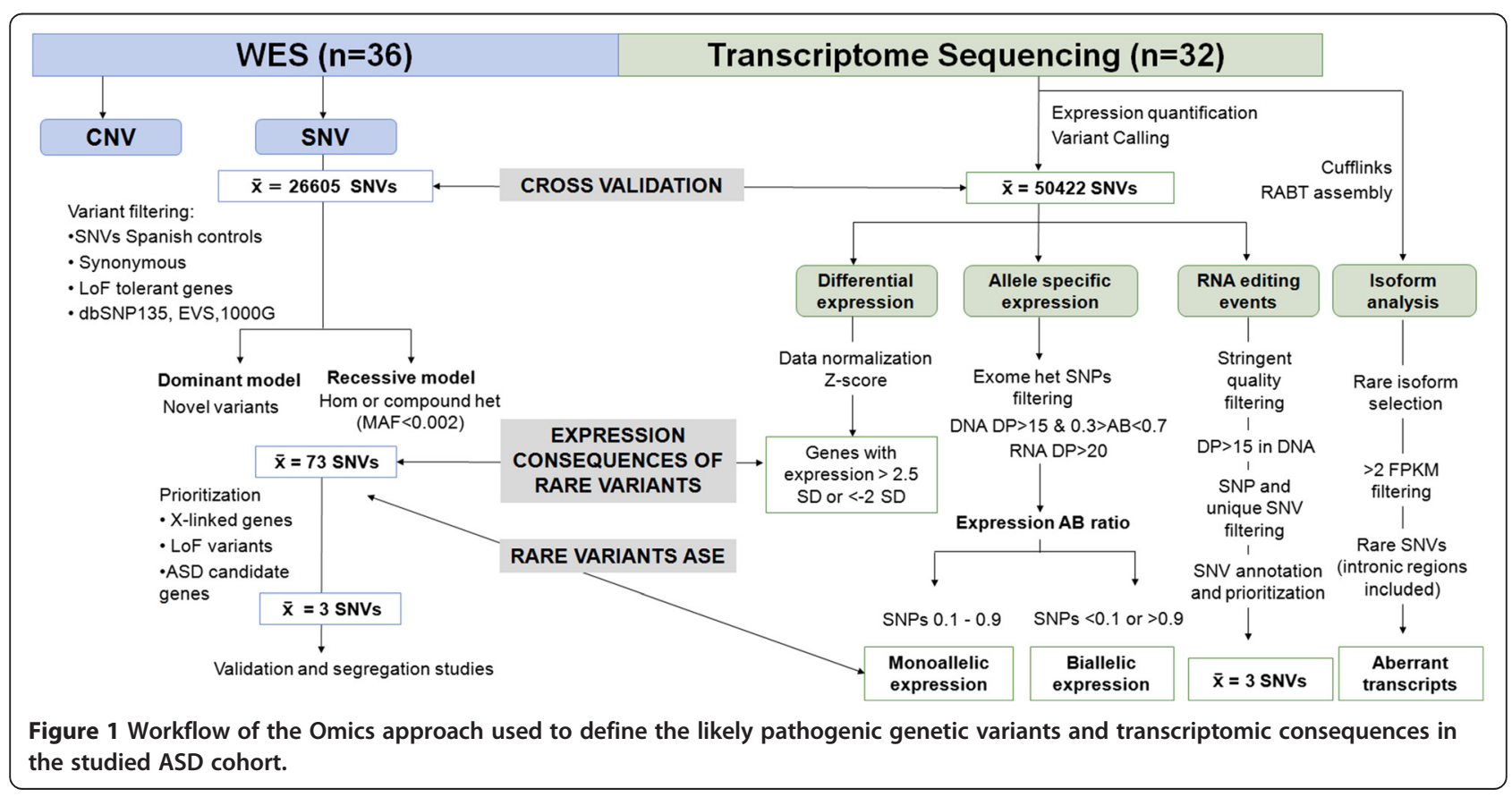


After filtering, considering only non-synonymous, frameshift, or splice site rare variants (MAF dominant $=0$; recessive $<0.002$ ), we selected a total of 2,626 calls, with an average of 73 rare events per patient (Figure 1). We classified the variants into missense and loss of function variants (LoF), a category which included splicing, non-sense, and frameshift mutations. Most rare variants were missense (91\%), while six per sample on average (9\%) were LoF (Additional file 1: Figure S1). The 2,626 rare variants were distributed in 2,205 genes, most of them carrying just one mutation, although some accumulated a high number of rare variants, with extreme cases such as MUC16 (MIM 606154) and TTN (MIM 188840) with 8 and 17 different mutations, respectively.

We considered likely pathogenic mutations all LoF variants, predicted damaging variants on the $\mathrm{X}$ chromosome or in autosomal candidate genes (according to the two main ASD databases, SFARI and AUTkB, see Additional file 2: Table S1) [33,34] and variants which involved a detectable change in the gene expression. To discriminate putative ASD pathogenic genes from non-related loci which accumulate LoF without any pathological consequence [24], we analyzed the distribution of this type of variants in the population with European ancestry described in the EVS $(n \sim 4,300)$, which contains exome sequencing data from individuals with unrelated disorders. We only considered as candidates the LoF variants that were found in genes with no LoF mutations in the general population. We finally selected 121 variants, with an average of 3 per patient. All novel variants absent in dbSNP137 $(n=109)$ were validated by Sequenom and/or Sanger sequencing, and segregation studies were performed using parental and other relatives' samples when available (Additional file 2: Table S2).

\section{Autosomal variants}

We studied the inheritance of 103 likely damaging variants (70 LoF and 33 missense) in candidate autosomal genes using parental samples. Only 5/103 rare variants (4.8\%) were de novo, and the remaining (95.2\%) were inherited from unaffected parents. De novo LoF mutations were detected in SCN2A (p.R583X), MED13L $[M I M$ 608771] (c.1708_1709delAG), KCNV1 [MIM 608164] (c.1391_1392delAT), and ADIPOR2 [MIM 607946] (p.R31X) in four different patients. Three of de novo LoF mutations are probably pathogenic, since they are predicted to result in truncated proteins. However, the de novo variant in ADIPOR2 found in a familial case (ASD_16) was not present in the affected sibling, excluding it as main ASD cause in this family although it could contribute as a modifier of the phenotype [35]. Additionally, we detected a de novo missense mutation affecting a highly conserved residue in CUL3 [MIM 603136] (p.H719R). Paternity was confirmed in all cases. Notably, none of these genes present a LoF variant in the general population. Detailed information about the phenotype of these patients is shown in Table 1 .

De novo non-sense SCN2A mutations have been already described in several cases of ASD [10,36-38]. The mutation we report is found in patient ASD_5; it results in a stop codon located after the first transmembrane domain, leading to a truncated protein with only one of the four transmembrane domains. The two basepair frameshift deletion in MED13L in patient ASD_11 generates a premature stop codon and a truncated protein lacking its main part. The frameshift mutation in KCNV1 in patient ASD_14 produces a stop codon shortly after, resulting in a prematurely truncated protein and abolishing the last cytoplasmatic domain. CUL3, mutated in ASD_7, has already been reported as ASD candidate, given that two de novo non-sense mutations were identified in previous work, a result that is highly unlikely by chance $[11,39]$.

All remaining variants were inherited from unaffected parents, suggesting either incomplete penetrance of these mutations or a contribution as an additive effect in those patients (Additional file 2: Table S2).

\section{X-linked variants}

We validated and performed segregation studies on all $\mathrm{X}$-linked variants that had not been previously reported or were found in candidate genes. We extended the study to the available healthy male family members to discard variants that did not segregate with the phenotype (Additional file 2: Table S3).

We found two X-linked mutations in MAOA (MIM 309850) and CDKL5 (MIM 300203). A splicing mutation (c.1438-2A > G) in MAOA was detected in the autistic proband (ASD_16) and later also in his brother diagnosed with pervasive developmental disorder not otherwise specified (PDD-NOS) (Table 1). The mother (mutation carrier) and maternal grandmother presented with a depressive and bipolar disorder, respectively. Unfortunately, no DNA was available from the grandmother to analyze the presence of the variant. The mutation led to aberrant splicing through the use of a cryptic splice site within intron 15 of $M A O A$ that was confirmed by RT-PCR of blood mRNA from the patients (Additional file 1: Figure S2). We also determined the levels of catecholamines and related metabolites in urine of these patients and their mother. These studies showed altered levels of several neurotransmitters in both affected brothers [increased serotonine $(2 \times)$, normetanephrine $(2.4 \times)$, and 5-HIAA $(0.45 \times)]$ and very mild in their mother (Additional file 2: Table S4), confirming the alteration of the monoamine metabolism.

A missense variant affecting a highly conserved residue (p.P647L) in the CDKL5 gene was also found in patient 
Table 1 Summary of the phenotypic features of ASD patients and relevant findings of the study

\begin{tabular}{lllllllll}
\hline Patient & $\begin{array}{l}\text { Birth } \\
\text { year }\end{array}$ & $\begin{array}{l}\text { Intellectual } \\
\text { disability }\end{array}$ & $\begin{array}{l}\text { ADIR } \\
\text { classification }\end{array}$ & $\begin{array}{l}\text { Seizure } \\
\text { episode }\end{array}$ & $\begin{array}{l}\text { Dysmorphic } \\
\text { features }\end{array}$ & $\begin{array}{l}\text { Functional } \\
\text { language }\end{array}$ & $\begin{array}{l}\text { Other medically } \\
\text { relevant } \\
\text { conditions }\end{array}$ & $\begin{array}{l}\text { Molecular } \\
\text { diagnosis }\end{array}$ \\
\hline ASD_1 $^{2} 1992$ & Severe & AUT & No & No & No & Obesity & CDKL5 (p. P647L) \\
ASD_2 & 1995 & Severe & PDD-NOS & No & No & No & Hypermetropia &
\end{tabular}

Specific ASD

overrepresented pathways

Agrin in postsynaptic differentiation

Elastic fiber formation Erk and pi-3 kinase for collagen binding in corneal epithelia

Integrin cell surface interactions

Molecules associated with elastic fibers

Ucalpain and friends in cell spread

$\begin{array}{lllllll}\text { ASD_3 } & 1993 & \text { Mild } & \text { AUT } & \text { Yes } & \text { No } & \text { No } \\ \text { ASD_4 } & 1995 & \text { Mild } & \text { AUT } & \text { Yes } & \text { No } & \text { No } \\ \text { ASD_5 } & 1997 & \text { Moderate } & \text { AUT } & \text { No } & \text { No } & \text { Yes } \\ \text { ASD_6 } & 1998 & \text { Mild } & \text { AUT } & \text { No } & \text { No } & \text { Yes } \\ \text { ASD_7 } & 1999 & \text { Mild } & \text { BS } & \text { No } & \text { Yes } & \text { Yes } \\ & & & & & \text { No } \\ \text { ASD_8 } & 1991 & \text { None } & \text { AUT } & \text { No } & \text { No } \\ \text { ASD_9 } & 2000 & \text { Moderate } & \text { AUT } & \text { No } & \text { No } & \text { No } \\ \text { ASD_10 } & 2000 & \text { Severe } & \text { AUT } & \text { No } & \text { No } & \text { No } \\ \text { ASD_11 } & 1999 & \text { Severe } & \text { AUT } & \text { No } & \text { Yes } & \text { No } \\ & & & & & & \text { No } \\ \text { ASD_12 } & 2002 & \text { Moderate } & \text { AUT } & \text { No } & \text { Yes } & \text { No } \\ \text { ASD_13 } & 2001 & \text { None } & \text { AUT } & \text { No } & \text { No } & \text { Yes }\end{array}$

No

No

Yes

Yes

Yes

Yes

No

No

No

No

Yes

Sleep disturbance, hypotonia, umbilical hernia ADHD disturbance ADHD ADHD

ADHD SCN2A (p.R583X)

Sleep CUL3 (p.H719R)

Sleep
disturbance,
hypotonia,
umbilical hernia
ADHD

$\begin{array}{ll} & \\ & \\ \text { ADHD } & \text { KCNV1 } \\ & \text { (c.1391_1392delAT) } \\ & \\ \begin{array}{l}\text { Mild psychosis, } \\ \text { aggressive } \\ \text { behavior }\end{array} & \text { MAOA } \\ & \text { (c.1438-2A }>\mathrm{G})\end{array}$

Integrin cell surface interactions

MED13L

(C.1708_1709delAG)
Elastic fiber formation Signaling events mediated by HDAC class II

Sumoylation by RanBP2 in transcriptional repression

\begin{tabular}{|c|c|c|c|c|c|c|c|c|}
\hline ASD_14 & 2000 & Severe & AUT & No & No & No & ADHD & $\begin{array}{l}\text { KCNV1 } \\
\text { (c.1391_1392delAT) }\end{array}$ \\
\hline ASD_15 & 2001 & Mild & AUT & Yes & Yes & Yes & & \\
\hline ASD_16 & 2001 & Moderate & AUT/PDD-NOS & Yes & No & Yes & $\begin{array}{l}\text { Mild psychosis, } \\
\text { aggressive } \\
\text { behavior }\end{array}$ & $\begin{array}{l}\text { MAOA } \\
(\text { c. } 1438-2 A>G)\end{array}$ \\
\hline
\end{tabular}

Beta1 integrin cell surface interactions Beta3 integrin cell surface interactions Integrin cell surface interactions 
Table 1 Summary of the phenotypic features of ASD patients and relevant findings of the study (Continued)

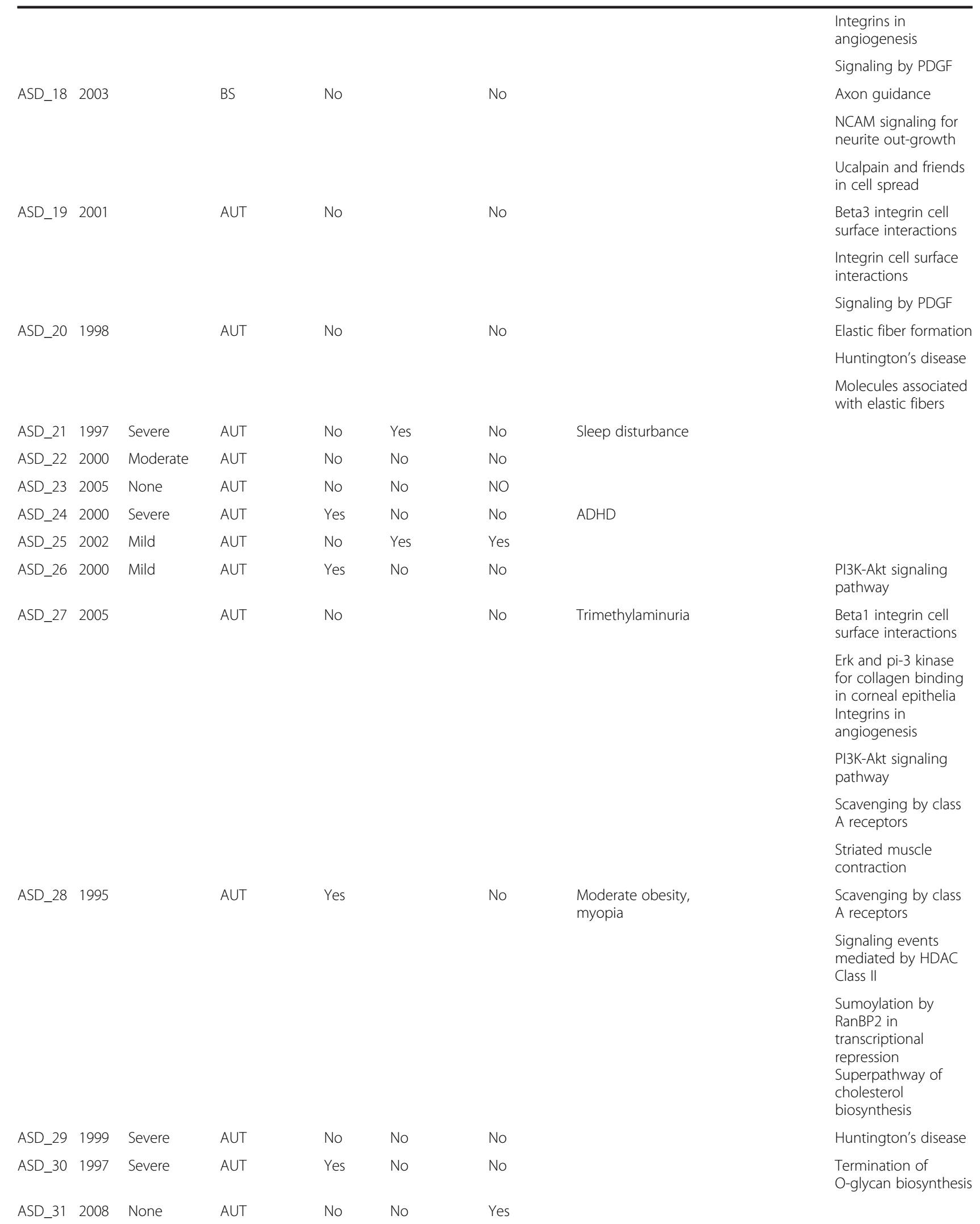


Table 1 Summary of the phenotypic features of ASD patients and relevant findings of the study (Continued)

\begin{tabular}{|c|c|c|c|c|c|c|c|c|c|}
\hline & & & & & & & & & $\begin{array}{l}\text { Striated muscle } \\
\text { contraction }\end{array}$ \\
\hline ASD_32 & 2004 & Mild & AUT & No & No & Yes & & & \\
\hline \multirow[t]{3}{*}{ ASD_33 } & 1990 & & AUT & No & & No & & & $\begin{array}{l}\text { Agrin in postsynaptic } \\
\text { differentiation }\end{array}$ \\
\hline & & & & & & & & & L1CAM interactions \\
\hline & & & & & & & & & $\begin{array}{l}\text { Ucalpain and friends } \\
\text { in cell spread }\end{array}$ \\
\hline \multirow[t]{2}{*}{ ASD_34 } & 2001 & & AUT & No & & Yes & & & $\begin{array}{l}\text { Superpathway of } \\
\text { cholesterol } \\
\text { biosynthesis }\end{array}$ \\
\hline & & & & & & & & & $\begin{array}{l}\text { Termination of } \\
\text { O-glycan biosynthesis }\end{array}$ \\
\hline \multirow[t]{4}{*}{ ASD_35 } & 2003 & & PDD-NOS & No & & Yes & & & Axon guidance \\
\hline & & & & & & & & & $\begin{array}{l}\text { Integrin cell surface } \\
\text { interactions }\end{array}$ \\
\hline & & & & & & & & & L1CAM interactions \\
\hline & & & & & & & & & $\begin{array}{l}\text { NCAM signaling for } \\
\text { neurite out-growth }\end{array}$ \\
\hline ASD_36 & 1999 & Severe & AUT & No & No & No & Macrocephaly & $\begin{array}{l}\text { PTEN } \\
(c .239-21 G>C)\end{array}$ & \\
\hline
\end{tabular}

ASD_1. Familial studies showed that the mutation was inherited from the mother and also present in the healthy sister but not in two healthy uncles. CDKL5 is a member of the Ser/Thr protein kinase family and encodes a phosphorylated protein with protein kinase activity. Mutations in this gene have been associated with X-linked infantile spasm (ISSX) and atypical Rett syndrome (RTT) [40-43].

We also carried out X chromosome inactivation (XCI) analyses on blood cell DNA from all 36 mothers of the probands, as an additional tool to predict putative pathogenicity of X-linked mutations. Six mothers were non-informative, 25 had a random XCI, and 5 showed a skewed XCI with ratios $>90 \%$. Among the XCI skewed mothers, only two presented rare variants in the $\mathrm{X}$ chromosome, being one also detected in a healthy uncle and the other inactivated preferentially the nontransmitted allele. In the CDKL5 mutation carriers, the $\mathrm{XCI}$ study was not informative in the mother but the proband's sister showed a selective inactivation of the maternally inherited mutated allele.

\section{Recurrently mutated genes in ASD}

We studied in detail the presence of recurrently mutated genes and the functional consequences resulting from the accumulation of rare genetic variants to identify common mechanisms involved in ASD etiology.

Since most putative pathogenic variants detected were inherited, we focused on genes mutated in more than one case of our ASD cohort. We found a total of 297 recurrently mutated genes, although most of them also showed a significant load of rare variants in the general population (EVS data). A total of 15 genes lacking LoF mutations in publicly available data from EVS were mutated with at least one LoF in more than one ASD case of our cohort (Additional file 2: Table S5). The list includes two genes with de novo variants in a patient and a missense mutation in the second patient ( SCN2A and $M E D 13 L)$. These variants cannot be fully penetrant mutations since they were inherited from healthy progenitors, but might behave as susceptibility factors in multifactorial or multiple-hit models. A proper association study with much larger sample size would be required to draw more clear conclusions about the pathogenicity of the recurrently mutated genes observed in this series.

\section{Common functional pathways affected by inherited rare mutations in ASD}

Previous studies suggest that, in addition to the relatively rare de novo and Mendelian forms, the etiology of ASD is mostly multifactorial with multiple genetic hits, mainly rare genetic variants in a few of hundreds of candidate genes $[10,11,44]$. To further define the potential pathogenic contribution to ASD of the inherited variants and identify common functional consequences, we conducted pathway enrichment analyses for each individual of our cohort excluding those with presumably monogenic forms ( $A S D_{-} 1$, $A S D \_5, A S D \_7, A S D \_11, A S D \_14, A S D \_16$, and $\left.A S D \_36\right)$ $(n=29)$ compared with 55 Spanish non-ASD controls. Using CPDB [45], we found 29 pathways overrepresented in ASD, 21 of them ASD-exclusive and absent in controls (Table 1). Interestingly, some of these pathways have also been previously related with the ASD phenotype, such as 
the PI3K/Akt signaling and the axon guidance [45-48]. We checked if the genes involved in monogenic cases are also involved in overrepresented pathways. The results show that three of the de novo mutated genes are part of overrepresented pathways. SCN2A is involved in Axon Guidance and L1CAM interaction pathways and PTEN is part of the PI3K-AKt and PDGF signaling pathways. These data underscore the role of those pathways in the pathogenesis of the ASD.

\section{Rare CNVs in ASD samples}

Although all patients had a previous molecular karyotype (either BAC, oligo, or SNP array), we analyzed copy number variants from exome data to identify small rare rearrangements that could have been previously missed. Using the XHMM algorithm and appropriate filtering, we detected a total of 11 rare CNVs (3 deletions and 8 duplications), ranging from 8.2 to $254 \mathrm{~Kb}$. All were validated by MLPA and segregation studies showed that all were inherited from an unaffected progenitor (Additional file 2: Table S6). One of these inherited duplications (patient ASD_9) at chr14q24.2 includes three genes (DCAF4, RBM25 [MIM 612427], ZFYVE1 [MIM 605471]) and partially disrupts two genes (PSEN1 [MIM 104311] and DPF3 [MIM 601672]). PSEN1 encodes a protein that regulates the processing of amyloid precursor protein (APP) and is mutated in familial forms of Alzheimer's disease while DPF3 function is associated with the BAF chromatin remodeling complex to promote transcription during development [49]. In addition, we detected two unrelated ASD individuals carrying a duplication of the X-Y pseudoautosomal ASMT [MIM 300015, 402500] previously associated with autism phenotype and sleep disturbance [50-52].

\section{Transcriptomic alterations in blood mRNA of ASD}

Good-quality blood transcriptome by RNAseq was available from 32 patients. Up to $64 \%$ of protein coding genes (14,114 of 22,057, according to Ensembl) were expressed at $>0.3$ FPKM, which is considered a robust threshold of gene expression [53]. No relevant differential transcriptomic pattern was evident in any group of samples by principal component analysis. We also compared individual samples against the rest in order to search for individual patterns of aberrant expression. The detectable variable genes per patient were unrelated and did not result in a common biological signature.

The integrative study of WES and RNAseq was limited to the almost $1 / 3(30 \%)$ of the positions identified as rare variants in exome data that had a depth of coverage $>10 \times$ in RNAseq, the established threshold for comparison. Out of those variants, $88 \%$ were also concordantly detected by RNAseq, showing high correlation between the two techniques and demonstrating the calling robustness.

\section{mRNA expression consequences of rare variation}

A total of 30 rare variants (1.7\% of all expressed) correlated with significant quantitative alterations in expression values of the same gene. All these variants were inherited from healthy progenitors (Additional file 2: Table S7). Among them, 6 were associated with decreased expression (z-score values $\leq 2$ ), whereas 24 were associated with overexpression (z-score values $>2.5$ ). Two rare mutations in SEMA6B [MIM 608873] correlated with $S E M A 6 B$ overexpression in two different samples (ASD_29 and ASD_33, Figure 2). Interestingly, ASD_29 carried two additional inherited candidate missense mutations in ANK3 [MIM 600465] and CREBBP [MIM 600140], which also correlated with overexpression of the mutated genes (Figure 2). Whereas the $C R E B B P$ variant was maternally inherited, the other two were inherited from the father, who committed suicide. We also detected a missense mutation (c.C1198T; p.P400S) previously described as a rare SNP in the MECP2 [MIM 300005] that correlated with higher expression of the gene $(\mathrm{z}$-score $=2.16)$ (Additional file 2: Table S7).

We also evaluated the expression changes of the 22 genes contained in the 11 rare CNVs detected in ASD patients. Only eight genes were expressed in blood at $>0.3$ FPKM, but we did not detect any significant outlier in expression (z-score values $\leq 2$ or $>2.5$ ). However, a global analysis comparing $\mathrm{z}$-score values between genes contained in all detectable deletions and duplications (a total of 26 expressed genes contained in any CNV), showed a tendency for higher expression values for duplications (mean $\mathrm{z}$-score $=0.43$ ) compared to deletions (mean $\mathrm{z}$ score $=-0.39$ ) (Additional file 1: Figure S3). Additionally, we detected 10 inherited rare LoF variants resulting in a premature stop codon associated with monoallelic expression of the wild-type allele, suggesting non-sense mediated mRNA decay (Additional file 2: Table S8).

We also searched for possible mRNA editing events in transcriptome data. After strict filtering process and manual curation of the variants in IGV, we did not detect any alteration indicative of mRNA editing. All mismatches between WES and RNAseq were either false negative WES calls or RNA sequencing errors located in the last position of the read or in a homopolymer region.

\section{Isoform-splicing analysis}

RNAseq technology offers the opportunity to identify not only quantitative but also qualitative changes, including novel isoform identification and accurate detection of biased allele expression, in comparison to previous technologies such as expression microarrays.

Isoform analysis by Cufflinks showed two aberrant transcripts due to rare genetic variants. The first was found in the PTEN and led to the insertion of an intronic fragment and the incorporation of six new amino 
A

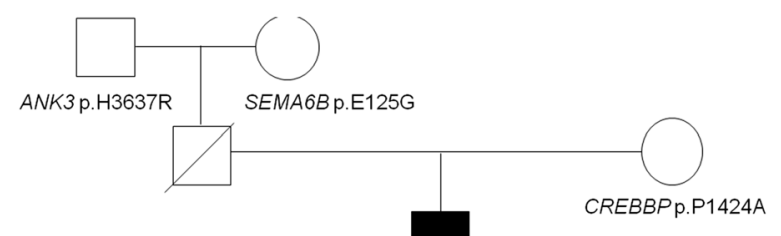

B
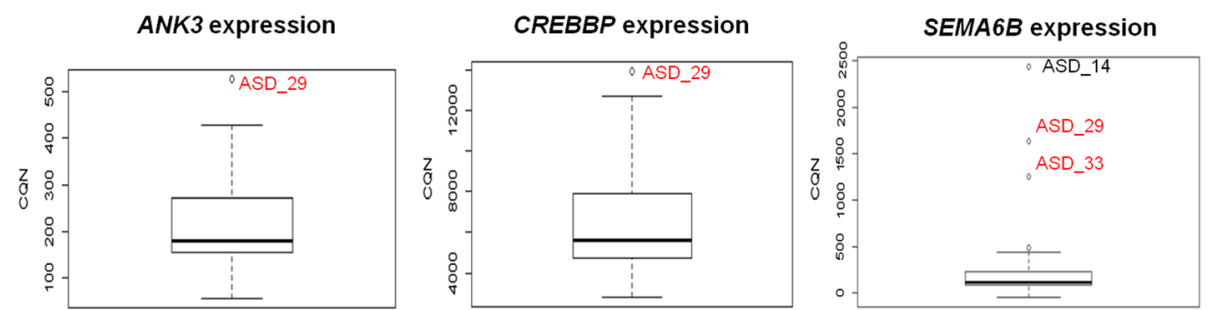

Figure 2 Quantitative changes in expression associated with rare variants. (A) Familial pedigree showing segregation of rare mutations. (B) Boxplot showing the distribution of expression values and expression outliers corresponding to the patients with rare mutations (in red).

acids to the protein. The new exon junction was found in close proximity to a deep intronic variant (c.239$21 \mathrm{G}>\mathrm{C}$ ) that generates a cryptic splice site. Segregation studies showed that it was de novo (Figure 3) and only present in patient ASD_36. The second aberrant transcript was found in POLR3C and retains an intron that generates a premature stop codon (ASD_2). The aberrant transcript is caused by a mutation that disrupts a splicing donor site $(c .104+1 G>A)$ inherited from the healthy father.

\section{Allele-specific expression}

To search for extreme imbalances of allelic expression or allele-specific expression, we studied 4,733 wellcovered genes with at least one heterozygous SNP not located in a known segmental duplication or a pseudogene. We found 4,426 genes (93\%) with biallelic and 81 (2\%) with monoallelic expression in all informative samples, while the expression was not homogenous among samples for the remaining 226 genes (5\%). Only three genes with consistent monoallelic expression in all samples (SNRPN [MIM 182279], ZDBF2, and ZNF597 [MIM 614685]) are known to be imprinted. On the other hand, we identified seven known imprinted genes with biallelic expression in blood, two of them previously reported (NAA60 [MIM 614246] and ZFAT [MIM 610931]) [54-56]. Regarding individual differences, we detected 78 genes with monoallelic expression in a single patient but biallelic in the remaining (at least five informative samples) excluding genes with biased expression $(A B$ ratio $>0.8$ or $<0.2$ ) in at least one sample (Additional file 1: Figure S4). We did not found any individual allelic imbalance in the opposite direction suggestive of loss of imprinting (Additional file 2: Table S9). Nine of the identified genes were previously described to have monoallelic expression in control samples [57-59]. The allelic expression imbalance was found in 26 of 32 samples, affecting between one and eight different genes with no regional clusters. None of these allelic expression imbalances were apparently related to cis-effects of the rare mutations or CNVs identified by WES. Interestingly, some of the altered genes have been associated to disease, such as MTOR [MIM 601231], FUS [MIM 137070], and TAF1C [MIM 604905].

\section{Discussion}

Autism spectrum disorders are a group of heterogeneous disorders with a strong genetic component but a complex genetic architecture. This complexity makes genetic diagnosis challenging, with a current diagnostic yield ranging from $15 \%$ to $30 \%$ [60,61]. Unbiased genome-wide molecular tools such as NGS, with a steadily lowering cost, have a proven efficacy, although they produce genetic and genomic information that cannot be properly interpreted yet. Here, we used WES and blood transcriptome by RNAseq in a selected group of males with idiopathic ASD to detect putative causal genetic variants of this complex disease. Segregation and recurrence analyses along with expression studies were used to better discriminate putatively pathogenic variants from innocuous rare variation.

WES identified several cases with likely monogenic forms of ASD, including four patients with de novo variants in strong candidate autosomal genes (11\%) and two patients with inherited X-linked mutations 


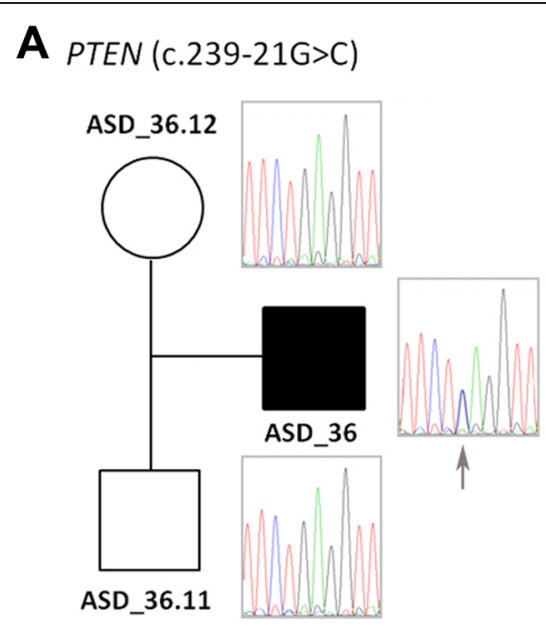

B POLR3C (c.104+1G>A)

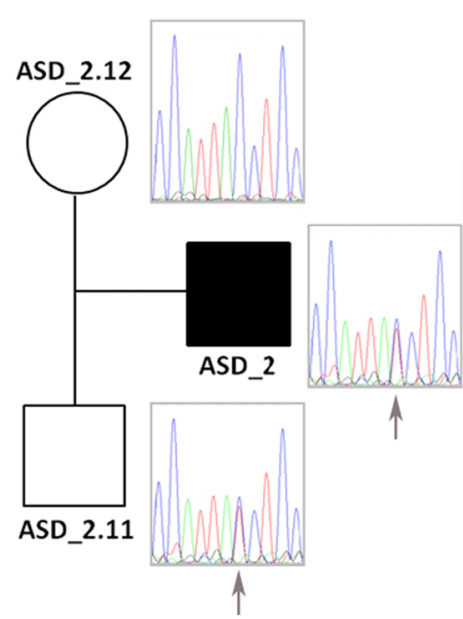

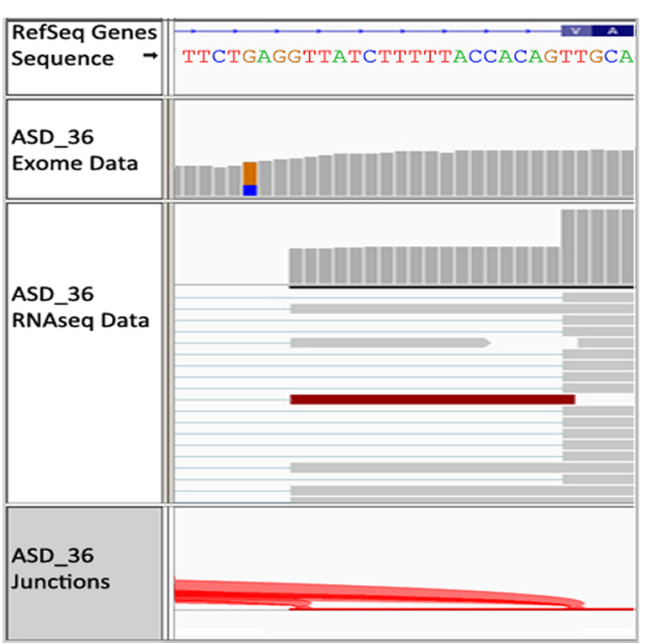

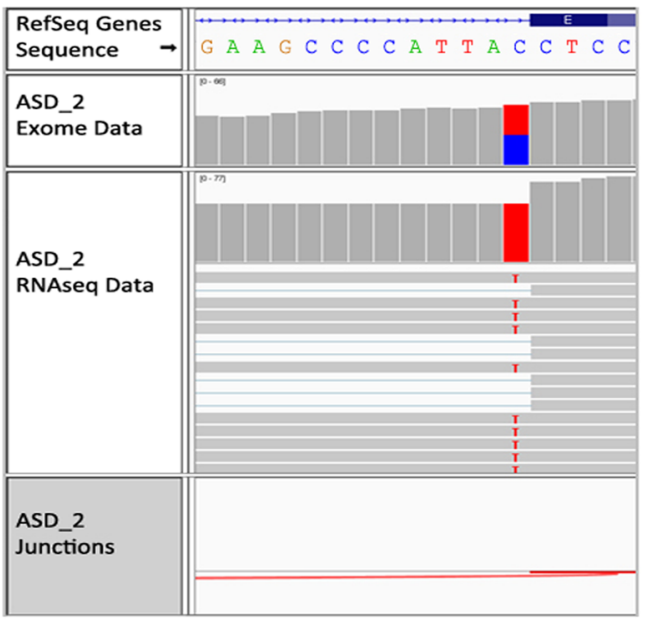

Figure 3 Segregation analysis by Sanger sequencing and Integrative Genomics Viewer (IGV) pileups in two trios. (A) de novo mutation (c.239-21G > C) found in the PTEN gene by exome sequencing and the aberrant transcript detected by transcriptome sequencing. (B) Paternal inherited mutation that disrupts a splicing donor site in POLR3C gene (c.104 + 1G > A) causing retention of an intron.

(5.6\%). Since our study did not include parental exome sequencing, our detection rate of monogenic cases may be underestimated. Autosomal LoF mutations were identified in $S C N 2 A, M E D 13 L$, and KCNV1. SCN2A is one of the few genes found recurrently mutated in unrelated patients with ASD and intellectual disability, which is unlikely to occur by chance $[10,36,37,62]$. MED $13 L$ was previously associated with intellectual disability and heart defects, and a de novo splicing mutation was described in an autistic patient $[13,63]$. Recently, de novo deletions affecting coding regions of $M E D 13 L$ were found in two girls presenting a phenotype very similar to the patient we report here, including facial dysmorphism, hypotonia, and development delay, along with ASD
[64]. Our findings are consistent with a role of MED13L in neurodevelopmental disorders. The third de novo and likely pathogenic variant affected $K C N V 1$, coding for a potassium channel subunit mainly expressed in the brain and involved in the regulation of two other potassium channels (KCNB1 and KCNB2). Defects in voltage-gated potassium channels have been associated with a variety of neuropsychiatric disorders, including bipolar disorder, schizophrenia, and ASD [12,65-67]. Therefore, our data suggest a role for potassium voltage-gated channels in the etiology of ASD. Finally, we detected a de novo missense mutation in the CUL3, which is other of the few recurrently de novo mutated genes in ASD patients $[11,39]$. 
Regarding X-linked mutations, we identified alterations in two genes (MAOA and CDKL5) previously associated with ASD and intellectual disability. We found a splicing mutation in $M A O A$ in a multiplex family with and X-linked pattern of inheritance, with two affected male siblings and a maternal history of psychiatric disease. $M A O A$ encodes the protein monoamine oxidase A, which degrades amine neurotransmitters such as dopamine, norepinephrine, and serotonin [68]. Both affected siblings had consistent biochemical alterations of the catecholamine pathway, very mild in their carrier mother, then supporting the pathogenicity of the mutation. A $M A O A$ truncating mutation was first described in a Dutch family in 1993, and recently, a second loss of function mutation was found in a family segregating ASD and behavioral problems [69,70]. Our work further strengthens the relation between $M A O A$ and ASD. The other X-linked mutation affected a highly conserved amino acid in CDKL5 and is predicted to be deleterious by different algorithms [71,72]. It was inherited from the healthy mother and also detected in the unaffected sister, who preferentially inactivated the mutated allele. Mutations in CKDL5 were reported in X-linked infantile spasms syndrome (ISSX) [MIM 308350], atypical Rett syndrome (RTT) [MIM 312750], and Angelman syndrome-like [MIM 105830] [40-42,73,74]. Since the phenotype of our patient is less severe than the one described in males with CDKL5 mutations, it is possible that the missense variant found in this patient is a hypomorphic allele with a milder effect. The biased $\mathrm{X}$ inactivation documented in the sister's proband could act as a protective factor in females explaining their unaffected status.

While the definition of the potential pathogenicity for ASD mutations could be relatively straightforward in de novo and Mendelian cases assuming full penetrance, a greater challenge is the classification of putatively pathogenic heterozygous mutations and rearrangements inherited from unaffected parents. These variants, presumably with incomplete penetrance and variable expression, are thought to contribute to disease risk in an oligogenic model with probable environmental contribution. One of the most common criteria to define potential pathogenicity is the recurrence of mutations in the same gene in unrelated patients. In our small cohort, we detected two additional patients with rare inherited missense mutations in genes with de novo LoF mutations in other cases (SCN2A and MED13L), suggesting that the inherited mutations could also be contributing to the disease. While amorphic alleles might be highly penetrant, hypomorphic missense mutations might have a milder effect just increasing the global burden of risk for ASD. Moreover, the study of common functional consequences of rare variation pointed towards a set of relevant pathways only overrepresented in ASD patients. Among these, there were the PI3K/Akt signaling and the axon guidance which were previously associated to ASD by linkage studies, rare CNVs, genomic mutations, and comorbid ASD conditions [45-48].

Using CNV detection tools on WES data, we identified small rearrangements that were missed by previous molecular karyotype in eight cases, all of them inherited from unaffected progenitors. Some of these rare CNVs could also contribute to the disease in a multiple-hit model, such as the duplications found in $A S M T$ as previously proposed [50-52]. Although we did not observe a significant effect on expression in the individual analysis of these rare CNVs, a global analysis showed a tendency for higher expression of genes in duplication type CNVs compared to those in deletions, as expected and described for other ASDrelated rearrangements $[20,75]$.

The incorporation of the peripheral blood transcriptomic data provided an important additive value to the identification of molecular biomarkers of ASD, leading to identification of additional mutations and transcriptional consequences. Despite that blood is not the ideal target tissue to study ASD, it is commonly used in neurodevelopmental disorders since it can be easily available for diagnostic testing [76]. In our study, approximately $30 \%$ of the rare variants were sufficiently expressed in blood, and $88 \%$ of these had a concordant calling in both techniques. By isoform analysis, we found an aberrant transcript in PTEN, due to a de novo intronic mutation that activates a cryptic splice site. The patient (ASD_36) presents macrocephaly, a feature that is consistently found in ASD patients with mutations in this gene (MIM 605309). Thus, transcriptome sequencing of blood cells was essential to achieve a diagnosis in an additional patient, reaching a final diagnosis yield of $19 \%$. Moreover, the integrative approach also enabled the identification of rare inherited variants with functional consequences that could contribute to the phenotype. As previously suggested, the joint study of genomic and transcriptomic data can be crucial to unravel the mechanism of complex diseases $[77,78]$. We detected alteration in expression levels in $1.7 \%$ of expressed rare variants, inherited in all cases. Overexpression of $M E C P 2$ was found in a patient who had a rare SNP variant in the same gene. Duplication of MECP2 causes a known duplication syndrome almost exclusively in males with moderate to severe intellectual disability. Overexpression of the gene in peripheral leucocytes was previously described in ASD patients [79] and was also related to aggressive social behavior in schizophrenia [80]. We also identified three rare mutations with concurring overexpression of candidate 
genes ANK3 [81,82], CREBBP [83,84], and SEMA6B [85] in a single patient. They were inherited from both progenitors and they could contribute to ASD in an additive manner. Additionally, we also detected monoallelic expression of the wild-type allele associated with ten rare inherited truncating mutations suggesting non-sense mediated decay (NMD) in which the functional haploinsufficiency could contribute to the phenotype such as the alteration in ALG9 [MIM 606941] and RIT1 [MIM 609591] previously involved in neuropshychiatric conditions [86-88]. Finally, allelespecific expression analysis revealed the alteration of 68 genes (or specific transcript variants) with monoallelic expression but no cis-element responsible for it. This phenomenon was found to be more common for autosomal and X-linked genes in ASD patients than in controls in the brain and other tissues [89,90]. Allelespecific expression can be caused by unidentified cis-acting elements, including genetic or genomic mutations in the promoter or regulatory regions and epigenetic marks. Thus, some of identified genes with allele-specific expression might contribute to ASD. In fact, two of them have been previously associated $(M T O R)$ [91] and/or are great candidates (FUS and TAF1C) $[92,93]$. Ongoing efforts to define the extent of expression variation in large numbers of healthy controls such as Geuvadis and GTEx will help to better clarify the deregulated genes found in individual patients that are related to disease.

\section{Conclusions}

In summary, our data reinforces the clinical utility of NGS in establishing the cause of ASD. Although transcriptome sequencing is limited by the genes expressed in the analyzed tissue, it has proven to be very useful in combination with WES. Their integration has determined the molecular defect compatible with highly penetrant monogenic ASD forms in 19\% of the studied cohort. Blood transcriptomic data also revealed functional correlations of genetic variants, including changes in splicing, expression levels, and allelic expression, as well as novel candidates with allelic imbalance. Considering the likely multifactorial etiology of most cases of ASD, data interpretation of the potentially deleterious variation inherited from unaffected progenitors still represents a challenge. Joint analysis of transcriptome sequencing and deregulated pathways by rare genetic variation can help to elucidate the genetic contribution of this complex disorder and define deregulated mechanisms. The identification of all genetic contributors in each patient will help to establish genotype-phenotype correlations, define specific disorders, and identify pathophysiologic mechanisms that can guide pharmacological approaches.

\section{Additional files}

Additional file 1: Figure S1. Distribution of WES variants per patient after filtering. Grey bars correspond to missense mutations, whereas red bars correspond to LoF mutations. Figure S2. A) Pedigree of the family ASD_16 compatible with X-linkage inheritance. B) Validation by Sanger sequencing of the hemizygous MAOA splicing mutation (c.1438-2A > G) in the proband and brother as well as the heterozygous mother. C) Schematic representation of the aberrant MAOA transcript detected in blood mRNA of the proband, generated by the use of a cryptic acceptor splice site in intron 15 of the gene. Figure S3. Distribution of z-score values among genes contained in all Copy Number Variant regions (CNVs) detected by XHMM in exome data. Figure S4. Boxplot showing distribution of RNA AB ratios for all informative SNPS located in genes showing ASE in ASD patients.

Additional file 2: Table S1. List of ASD candidate genes. Table S2. Variants selected for validation and segregation after filtering. Table S3. X-linked variants selected for validation and segregation studies. Table S4. Cathecolamine and metabolites in 24 hours urine. Table S5. Recurrently mutated genes with no LoF reported in EVS. Table S6. Rare CNVs selected for validation and segregation. Table S7. Rare mutations with changes in expression levels. Table S8. Detected nonsense mediated decay events. Table S9. Genes with allele specific expression.

\section{Abbreviations}

ADHD: attention deficit hyperactivity disorder; ADI-R: Autism Diagnostic Interview Revised; ASD: autism spectrum disorders; ASE: allele-specific expression; CNV: copy number variants; CPDB: ConsensusPathDB; DGV: Database of Genomic Variants; DP: depth of coverage; DSMIV: Diagnostic and Statistical Manual of Mental Disorders; EVS: Exome Variant Server; FPKM: fragments per kilobase of transcript per million mapped reads; GATK: Genome Analysis Toolkit; IGV: Integrative Genome Viewer; ISSX: X-linked infantile spasm syndrome; LoF: loss of function; MAF: minimum allele frequencing; MLPA: multiplex ligation probe amplification; NGS: nextgeneration sequencing; PAR1: pseudoautosomal region 1; PBMCs: peripheral blood mononuclear cells; PDD-NOS: pervasive developmental disorder not otherwise specified; RTT: Rett syndrome; SNV: single nucleotide variant; WES: whole-exome sequencing; $\mathrm{XCl}$ : X chromosome inactivation.

\section{Competing interests}

Benjamín Rodríguez-Santiago and Luis A. Pérez-Jurado are currently employee and scientific advisor, respectively, of qGenomics SL. The authors declare no competing financial and non-financial conflicts of interests.

\section{Authors' contributions}

MCS participated in the performance of molecular genetic assays, carried out data interpretation, and drafted the manuscript. BRS performed the bioinformatic analyses and helped to draft the manuscript. AH participated in genetic studies and revised the manuscript. JS participated in the bioinformatic analyses. MR participated in the molecular studies. GAL, MdC, $B G, E G$, and MPB carried out the clinical evaluation of the patients. AG participated in the bioinformatic analyses. GA participated in the whole-exome sequencing process. LPJ conceived the study and participated in the design and data interpretation, and helped in drafting the manuscript. IC conceived the study and participated in the design, coordination, and data interpretation and drafted the manuscript. All authors read and approved the manuscript.

\section{Acknowledgements}

We would like to thank the patients and their families for their support. This work was funded by grants from the Spanish Ministry of Health (FIS PI1002512, PI1302481, and PI1300823 cofunded by FEDER), Fundación Alicia Koplowitz and Generalitat de Catalunya (2014SGR1468). The 'Medical Genome Project' is a joint initiative of the Consejería de Salud de la Junta de Andalucía and Roche, supported by the 'Programa Nacional de Proyectos de investigación Aplicada', I + D + i 2008, 'Subprograma de actuaciones Científicas y Tecnológicas en Parques Científicos y Tecnológicos' (ACTEPARQ 2009) and FEDER. The CIBER de Enfermedades Raras is an initiative of the ISCIII. CDTI FEDER-Innterconecta EXP00052887/ITC-20111037. 


\section{Author details}

${ }^{1}$ Department of Experimental and Health Sciences, Universitat Pompeu Fabra, C/Doctor Aiguader 88, 422, Barcelona 08003, Spain. ${ }^{2}$ Hospital del Mar Research Institute (IMIM), C/Doctor Aiguader 88, Barcelona 08003, Spain. ${ }^{3}$ Centro de Investigación Biomédica en Red de Enfermedades Raras (CIBER-ER), C/ Monforte de Lemos 3-5, Madrid 28029, Spain. ${ }^{4}$ Quantitative Genomic Medicine Laboratories (qGenomics), C/Doctor Aiguader 88, 422, Barcelona 08003, Spain. ${ }^{5}$ Medical Genome Project, Genomics and Bioinformatics Platform of Andalusia (GBPA), C/Albert Einstein, Cartuja Scientific and Technology Park, INSUR Builiding, Sevilla 41092, Spain. ${ }^{6}$ Pediatric Neurology, Hospital del Mar, Passeig Marítim 25-29, Barcelona 08003, Spain. ${ }^{7}$ Servicio de Genética, Hospital Vall d'Hebron, Passeig Vall d'Hebron, 119-129, Barcelona 08015, Spain. ${ }^{8}$ Genetics Service, BioCruces Health Research Institute, Hospital Universitario Cruces, Plaza de Cruces 12, Barakaldo, Bizkaia 48093, Spain. ${ }^{9}$ Pediatrics Service, Corporació Sanitària Parc Taulí, Parc Taulí 1, Sabadell 08208, Spain. ${ }^{10}$ Pediatric Neurology, Hospital de Txagorritxu, C/José de Atxotegui s/n, Victoria-Gasteiz 01009, Spain. ${ }^{11}$ Department of Genetics, Reproduction and Fetal Medicine, Institute of Biomedicine of Seville (IBIS), University Hospital Virgen del Rocío/CSIC/ University of Seville, Avda Manuel Siurot s/n, Sevilla 41013, Spain.

\section{Received: 4 December 2014 Accepted: 19 March 2015} Published online: 15 April 2015

\section{References}

1. CDC. Prevalence of autism spectrum disorders-autism and developmenta disabilities monitoring network, 14 sites, United States, 2008. MMWR Surveill Summ. 2012;61:1-19.

2. Gurrieri F. Working up autism: the practical role of medical genetics. Am J Med Genet C: Semin Med Genet. 2012;160C:104-10.

3. Fombonne E. The prevalence of autism. JAMA. 2003;289:87-9.

4. Smalley SL. Genetic influences in childhood-onset psychiatric disorders: autism and attention-deficit/hyperactivity disorder. Am J Hum Genet. 1997;60:1276-82.

5. Marco EJ, Skuse DH. Autism-lessons from the X chromosome. Soc Cogn Affect Neurosci. 2006;1:183-93.

6. Duchan E, Patel DR. Epidemiology of autism spectrum disorders. Pediatr Clin North Am. 2012;59:27-43.

7. Gupta AR, State MW. Recent advances in the genetics of autism. Bio Psychiatry. 2007;61:429-37.

8. Beaudet AL. Autism: highly heritable but not inherited. Nat Med. 2007;13:534-6

9. O'Roak BJ, Deriziotis P, Lee C, Vives L, Schwartz JJ, Girirajan S, et al. Exome sequencing in sporadic autism spectrum disorders identifies severe de novo mutations. Nat Genet. 2011:43:585-9.

10. Sanders SJ, Murtha MT, Gupta AR, Murdoch JD, Raubeson MJ, Willsey AJ, et al. De novo mutations revealed by whole-exome sequencing are strongly associated with autism. Nature. 2012;485:237-41.

11. O'Roak BJ, Vives L, Girirajan S, Karakoc E, Krumm N, Coe BP, et al. Sporadic autism exomes reveal a highly interconnected protein network of de novo mutations. Nature. 2012;485:246-50.

12. Neale BM, Kou Y, Liu L, Ma'ayan A, Samocha KE, Sabo A, et al. Patterns and rates of exonic de novo mutations in autism spectrum disorders. Nature. 2012;485:242-5.

13. Iossifov I, Ronemus M, Levy D, Wang Z, Hakker I, Rosenbaum J, et al. De novo gene disruptions in children on the autistic spectrum. Neuron. 2012;74:285-99.

14. Iossifov I, O'Roak BJ, Sanders SJ, Ronemus M, Krumm N, Levy D, et al. The contribution of de novo coding mutations to autism spectrum disorder. Nature. 2014;515:216-21.

15. Chahrour MH, Yu TW, Lim ET, Ataman B, Coulter ME, Hill RS, et al. Whole-exome sequencing and homozygosity analysis implicate depolarization-regulated neuronal genes in autism. PLoS Genet. 2012;8:e1002635.

16. Shi L, Zhang X, Golhar R, Otieno FG, He M, Hou C, et al. Whole-genome sequencing in an autism multiplex family. Mol Autism. 2013;4:8.

17. Toma C, Torrico B, Hervas A, Valdes-Mas R, Tristan-Noguero A, Padillo V, et al. Exome sequencing in multiplex autism families suggests a major role for heterozygous truncating mutations. Mol Psychiatry. 2013;19:784-90.

18. De Rubeis S, He X, Goldberg AP, Poultney CS, Samocha K, Ercument Cicek $A$, et al. Synaptic, transcriptional and chromatin genes disrupted in autism. Nature. 2014;515:209-15.
19. Liu L, Sabo A, Neale BM, Nagaswamy U, Stevens C, Lim E, et al. Analysis of rare, exonic variation amongst subjects with autism spectrum disorders and population controls. PLoS Genet. 2013;9:e1003443.

20. Luo R, Sanders SJ, Tian Y, Voineagu I, Huang N, Chu SH, et al. Genome-wide transcriptome profiling reveals the functional impact of rare de novo and recurrent CNVs in autism spectrum disorders. Am J Hum Genet. 2012;91:38-55.

21. Homer N, Merriman B, Nelson SF. BFAST: an alignment tool for large scale genome resequencing. PLoS One. 2009;4:e7767.

22. McKenna AHM, Banks E, Sivachenko A, Cibulskis K, Kernytsky A, Garimella K, et al. The Genome Analysis Toolkit: a MapReduce framework for analyzing next-generation DNA sequencing data. Genome Res. 2010;20(9):1297-303.

23. González-Pérez A, Lopez-Bigas N. Improving the assessment of the outcome of nonsynonymous SNVs with a consensus deleteriousness score. Am J Hum Genet. 2011:88:440-9.

24. MacArthur DG, Balasubramanian S, Frankish A, Huang N, Morris J, Walter K et al. A systematic survey of loss-of-function variants in human protein-coding genes. Science. 2012;335:823-8.

25. Thorvaldsdottir H, Robinson JT, Mesirov JP. Integrative Genomics Viewer (IGV): high-performance genomics data visualization and exploration. Brief Bioinform. 2013;14:178-92.

26. Poultney CS, Goldberg AP, Drapeau E, Kou Y, Harony-Nicolas H, Kajiwara Y, et al. Identification of small exonic CNV from whole-exome sequence data and application to autism spectrum disorder. Am J Hum Genet. 2013;93:607-19.

27. Pegoraro E, Schimke RN, Arahata K, Hayashi Y, Stern H, Marks H, et al. Detection of new paternal dystrophin gene mutations in isolated cases of dystrophinopathy in females. Am J Hum Genet. 1994;54:989-1003.

28. Jang SJ, Mao L. Methylation patterns in human androgen receptor gene and clonality analysis. Cancer Res. 2000;60:864-6.

29. Kim D, Pertea G, Trapnell C, Pimentel H, Kelley R, Salzberg SL. TopHat2: accurate alignment of transcriptomes in the presence of insertions, deletions and gene fusions. Genome Biol. 2013;14:R36.

30. Langmead B, Trapnell C, Pop M, Salzberg SL. Ultrafast and memory-efficient alignment of short DNA sequences to the human genome. Genome Biol. 2009;10:R25

31. Trapnell C, Williams BA, Pertea G, Mortazavi A, Kwan G, van Baren MJ, et al. Transcript assembly and quantification by RNA-Seq reveals unannotated transcripts and isoform switching during cell differentiation. Nat Biotechnol. 2010:28:511-5.

32. Anders S, Pyl PT, Huber W. HTSeq-a Python framework to work with high-throughput sequencing data. Bioinformatics. 2015;31(2):166-9.

33. Banerjee-Basu S, Packer A. SFARI Gene: an evolving database for the autism research community. Dis Model Mech. 2010;3:133-5.

34. Xu LM, Li JR, Huang Y, Zhao M, Tang X, Wei L. AutismKB: an evidence-based knowledgebase of autism genetics. Nucleic Acids Res. 2012;40:D1016-22.

35. Adams DR, Yuan $H$, Holyoak T, Arajs KH, Hakimi P, Markello TC, et al. Three rare diseases in one Sib pair: RAI1, PCK1, GRIN2B mutations associated with Smith-Magenis Syndrome, cytosolic PEPCK deficiency and NMDA receptor glutamate insensitivity. Mol Gen Metab. 2014;113:161-70.

36. Rauch A, Wieczorek D, Graf E, Wieland T, Endele S, Schwarzmayr T, et al. Range of genetic mutations associated with severe non-syndromic sporadic intellectual disability: an exome sequencing study. Lancet. 2012;380:1674-82

37. de Ligt J, Willemsen MH, van Bon BW, Kleefstra T, Yntema HG, Kroes T, et al. Diagnostic exome sequencing in persons with severe intellectual disability. N Engl J Med. 2012;367:1921-9.

38. Tavassoli T, Kolevzon A, Wang AT, Curchack-Lichtin J, Halpern D, Schwartz L, et al. De novo SCN2A splice site mutation in a boy with autism spectrum disorder. BMC Med Genet. 2014;15:35.

39. Kong A, Frigge ML, Masson G, Besenbacher S, Sulem P, Magnusson G, et al. Rate of de novo mutations and the importance of father's age to disease risk. Nature. 2012;488:471-5.

40. Liang JS, Shimojima K, Takayama R, Natsume J, Shichiji M, Hirasawa K, et al. CDKL5 alterations lead to early epileptic encephalopathy in both genders. Epilepsia. 2011;52:1835-42.

41. Bartnik M, Derwinska K, Gos M, Obersztyn E, Kolodziejska KE, Erez A, et al. Early-onset seizures due to mosaic exonic deletions of CDKL5 in a male and two females. Genet Med. 2011;13:447-52.

42. Weaving LS, Christodoulou J, Williamson SL, Friend KL, McKenzie OL, Archer $\mathrm{H}$, et al. Mutations of CDKL5 cause a severe neurodevelopmental disorder with infantile spasms and mental retardation. Am J Hum Genet. 2004;75:1079-93. 
43. Russo S, Marchi M, Cogliati F, Bonati MT, Pintaudi M, Veneselli E, et al. Novel mutations in the CDKL5 gene, predicted effects and associated phenotypes. Neurogenetics. 2009;10:241-50.

44. Sanders SJ, Ercan-Sencicek AG, Hus V, Luo R, Murtha MT, Moreno-De-Luca $D$, et al. Multiple recurrent de novo CNVs, including duplications of the 7q11.23 Williams syndrome region, are strongly associated with autism. Neuron. 2011;70:863-85.

45. Kamburov A, Pentchev K, Galicka H, Wierling $\mathrm{C}$, Lehrach $\mathrm{H}$, Herwig R. ConsensusPathDB: toward a more complete picture of cell biology. Nucleic Acids Res. 2011;39:D712-7.

46. Cusco I, Medrano A, Gener B, Vilardell M, Gallastegui F, Villa O, et al. Autismspecific copy number variants further implicate the phosphatidylinositol signaling pathway and the glutamatergic synapse in the etiology of the disorder. Hum Mol Gen. 2009;18:1795-804.

47. Suda S, Iwata K, Shimmura C, Kameno Y, Anitha A, Thanseem I, et al. Decreased expression of axon-guidance receptors in the anterior cingulate cortex in autism. Mol Autism. 2011:2:14.

48. Engle EC. Human genetic disorders of axon guidance. Cold Spring Harb Perspect Biol. 2010;2:a001784

49. Zeng L, Zhang Q, Li S, Plotnikov AN, Walsh MJ, Zhou MM. Mechanism and regulation of acetylated histone binding by the tandem PHD finger of DPF3b. Nature. 2010;466:258-62.

50. Cai G, Edelmann L, Goldsmith JE, Cohen N, Nakamine A, Reichert JG, et al. Multiplex ligation-dependent probe amplification for genetic screening in autism spectrum disorders: efficient identification of known microduplications and identification of a novel microduplication in ASMT. BMC Med Genomics. 2008;1:50.

51. Melke J, Goubran Botros H, Chaste P, Betancur C, Nygren G, Anckarsater H, et al. Abnormal melatonin synthesis in autism spectrum disorders. Mol Psychiatry. 2008;13:90-8.

52. Wang L, Li J, Ruan Y, Lu T, Liu C, Jia M, et al. Sequencing ASMT identifies rare mutations in Chinese Han patients with autism. PLoS One. 2013;8:e53727

53. Ramskold D, Wang ET, Burge CB, Sandberg R. An abundance of ubiquitously expressed genes revealed by tissue transcriptome sequence data. PLoS Comput Biol. 2009:5:e1000598.

54. Frost JM, Monk D, Stojilkovic-Mikic T, Woodfine K, Chitty LS, Murrell A, et al. Evaluation of allelic expression of imprinted genes in adult human blood. PLoS One. 2010;5:e13556.

55. Barbaux S, Gascoin-Lachambre G, Buffat C, Monnier P, Mondon F, Tonanny $M B$, et al. A genome-wide approach reveals novel imprinted genes expressed in the human placenta. Epigenetics. 2012;7:1079-90.

56. Nakabayashi K, Trujillo AM, Tayama C, Camprubi C, Yoshida W, Lapunzina P, et al. Methylation screening of reciprocal genome-wide UPDs identifies novel human-specific imprinted genes. Hum Mol Gen. 2011;20:3188-97.

57. Song MY, Kim HE, Kim S, Choi IH, Lee JK. SNP-based large-scale identification of allele-specific gene expression in human B cells. Gene. 2012;493:211-8.

58. Pant PV, Tao H, Beilharz EJ, Ballinger DG, Cox DR, Frazer KA. Analysis of allelic differential expression in human white blood cells. Genome Res. 2006:16:331-9.

59. Pollard KS, Serre D, Wang X, Tao H, Grundberg E, Hudson TJ, et al. A genome-wide approach to identifying novel-imprinted genes. Hum Genet. 2008;122:625-34

60. Miles JH. Autism spectrum disorders-a genetics review. Genet Med. 2011;13:278-94

61. Schaefer GB, Mendelsohn NJ. Clinical genetics evaluation in identifying the etiology of autism spectrum disorders. Genet Med. 2008;10:301-5.

62. Jiang YH, Yuen RK, Jin X, Wang M, Chen N, Wu X, et al. Detection of clinically relevant genetic variants in autism spectrum disorder by whole-genome sequencing. Am J Hum Genet. 2013;2:249-63.

63. Muncke $\mathrm{N}$, Jung $\mathrm{C}$, Rudiger $\mathrm{H}$, Ulmer $\mathrm{H}$, Roeth $\mathrm{R}$, Hubert $\mathrm{A}$, et al. Missense mutations and gene interruption in PROSIT240, a novel TRAP240-like gene, in patients with congenital heart defect (transposition of the great arteries) Circulation. 2003;108:2843-50

64. Asadollahi R, Oneda B, Sheth F, Azzarello-Burri S, Baldinger R, Joset P, et al. Dosage changes of MED13L further delineate its role in congenital heart defects and intellectual disability. Eur J Hum Genet. 2013:21:110-4.

65. Laumonnier F, Roger S, Guerin P, Molinari F, M'Rad R, Cahard D, et al. Association of a functional deficit of the BKCa channel, a synaptic regulator of neuronal excitability, with autism and mental retardation. Am J Psychiatry. 2006;163:1622-9.

66. Sicca F, Imbrici P, D'Adamo MC, Moro F, Bonatti F, Brovedani P, et al. Autism with seizures and intellectual disability: possible causative role of gain-offunction of the inwardly-rectifying K+ channel Kir4.1. Neurobiol Dis. 2011:43:239-47.

67. Smolin B, Karry R, Gal-Ben-Ari S, Ben-Shachar D. Differential expression of genes encoding neuronal ion-channel subunits in major depression, bipolar disorder and schizophrenia: implications for pathophysiology. Int J Neuropsychopharmacol. 2012;15:869-82.

68. Ozelius L, Hsu YP, Bruns G, Powell JF, Chen S, Weyler W, et al. Human monoamine oxidase gene (MAOA): chromosome position (Xp21-p11) and DNA polymorphism. Genomics. 1988;3:53-8.

69. Brunner HG, Nelen M, Breakefield XO, Ropers HH, van Oost BA. Abnormal behavior associated with a point mutation in the structural gene for monoamine oxidase A. Science. 1993;262:578-80.

70. Piton A, Poquet H, Redin C, Masurel A, Lauer J, Muller J, et al. 20 ans apres: a second mutation in MAOA identified by targeted high-throughput sequencing in a family with altered behavior and cognition. Eur J Hum Genet. 2014;22:776-83

71. Kumar P, Henikoff S, Ng PC. Predicting the effects of coding nonsynonymous variants on protein function using the SIFT algorithm. Nat Protoc. 2009:4:1073-81.

72. Adzhubei IA, Schmidt S, Peshkin L, Ramensky VE, Gerasimova A, Bork P, et al. A method and server for predicting damaging missense mutations. Nat Methods. 2010;7:248-9.

73. Fehr S, Wilson M, Downs J, Williams S, Murgia A, Sartori S, et al. The CDKL5 disorder is an independent clinical entity associated with early-onset encephalopathy. Eur J Hum Genet. 2013;21:266-73.

74. Tao J, Van Esch H, Hagedorn-Greiwe M, Hoffmann K, Moser B, Raynaud M, et al. Mutations in the X-linked cyclin-dependent kinase-like 5 (CDKL5/STK9) gene are associated with severe neurodevelopmental retardation. Am J Hum Genet. 2004;75:1149-54

75. Mehta D, Iwamoto K, Ueda J, Bundo M, Adati N, Kojima T, et al. Comprehensive survey of CNVs influencing gene expression in the human brain and its implications for pathophysiology. Neurosci Res. 2014:79:22-33.

76. Tylee DS, Kawaguchi DM, Glatt SJ. On the outside, looking in: a review and evaluation of the comparability of blood and brain "-omes". Am J Med Genet B Neuropsychiatr Genet. 2013;162B:595-603.

77. Kukurba KR, Zhang R, Li X, Smith KS, Knowles DA, How Tan M, et al. Allelic expression of deleterious protein-coding variants across human tissues. PLoS Genet. 2014;10:e1004304.

78. Zhao Q, Kirkness EF, Caballero OL, Galante PA, Parmigiani RB, Edsall L, et al. Systematic detection of putative tumor suppressor genes through the combined use of exome and transcriptome sequencing. Genome Biol. 2010;11:R114.

79. Kuwano Y, Kamio Y, Kawai T, Katsuura S, Inada N, Takaki A, et al. Autismassociated gene expression in peripheral leucocytes commonly observed between subjects with autism and healthy women having autistic children. PLoS One. 2011;6:e24723.

80. Tantra M, Hammer C, Kastner A, Dahm L, Begemann M, Bodda C, et al Mild expression differences of MECP2 influencing aggressive social behavior. EMBO Mol Med. 2014;6:662-84

81. Allen AS, Berkovic SF, Cossette P, Delanty N, Dlugos D, Eichler EE, et al. De novo mutations in epileptic encephalopathies. Nature. 2013:501:217-21.

82. Bi C, Wu J, Jiang T, Liu Q, Cai W, Yu P, et al. Mutations of ANK3 identified by exome sequencing are associated with autism susceptibility. Hum Mutat. 2012;33:1635-8.

83. Barnby G, Abbott A, Sykes N, Morris A, Weeks DE, Mott R, et al. Candidate-gene screening and association analysis at the autism-susceptibility locus on chromosome 16p: evidence of association at GRIN2A and ABAT. Am J Hum Genet. 2005;76:950-66.

84. Petrij F, Giles RH, Dauwerse HG, Saris JJ, Hennekam RC, Masuno M, et al. Rubinstein-Taybi syndrome caused by mutations in the transcriptional co-activator CBP. Nature. 1995:376:348-51.

85. Andermatt I, Wilson NH, Bergmann T, Mauti O, Gesemann M, Sockanathan $\mathrm{S}$, et al. Semaphorin $6 \mathrm{~B}$ acts as a receptor in post-crossing commissural axon guidance. Development. 2014;19:3709-20.

86. Baysal BE, Willett-Brozick JE, Badner JA, Corona W, Ferrell RE, Nimgaonkar VL, et al. A mannosyltransferase gene at $11 \mathrm{q} 23$ is disrupted by a translocation 
breakpoint that co-segregates with bipolar affective disorder in a small family. Neurogenetics. 2002;4:43-53.

87. Aoki Y, Niihori T, Banjo T, Okamoto N, Mizuno S, Kurosawa K, et al. Gain-offunction mutations in RIT1 cause Noonan syndrome, a RAS/MAPK pathway syndrome. Am J Hum Genet. 2013;93:173-80.

88. Garbett K, Ebert PJ, Mitchell A, Lintas C, Manzi B, Mirnics K, et al. Immune transcriptome alterations in the temporal cortex of subjects with autism. Neurobiol Dis. 2008:30:303-11.

89. Ben-David E, Shohat S, Shifman S. Allelic expression analysis in the brain suggests a role for heterogeneous insults affecting epigenetic processes in autism spectrum disorders. Hum Mol Gen. 2014;23:4111-24.

90. Ben-David E, Granot-Hershkovitz E, Monderer-Rothkoff G, Lerer E, Levi S, Yaari $\mathrm{M}$, et al. Identification of a functional rare variant in autism using genome-wide screen for monoallelic expression. Hum Mol Gen. 2011;20:3632-41.

91. Hoeffer CA, Klann E. mTOR signaling: at the crossroads of plasticity, memory and disease. Trends Neurosci. 2010;33:67-75.

92. Stamova BS, Tian Y, Nordahl CW, Shen MD, Rogers S, Amaral DG, et al. Evidence for differential alternative splicing in blood of young boys with autism spectrum disorders. Mol Autism. 2013;4:30.

93. Anney R, Klei L, Pinto D, Regan R, Conroy J, Magalhaes TR, et al. A genome-wide scan for common alleles affecting risk for autism. Hum Mol Gen. 2010;19:4072-82.

\section{Submit your next manuscript to BioMed Central and take full advantage of:}

- Convenient online submission

- Thorough peer review

- No space constraints or color figure charges

- Immediate publication on acceptance

- Inclusion in PubMed, CAS, Scopus and Google Scholar

- Research which is freely available for redistribution 